Review

\title{
Nano- and Microdelivery Systems for Marine Bioactive Lipids
}

\section{David M. Pereira, Patrícia Valentão and Paula B. Andrade *}

REQUIMTE/Laboratory of Pharmacognosy, Department of Chemistry, Faculty of Pharmacy, University of Porto, Rua de Jorge Viterbo Ferreira $n^{\circ} 228$, 4050-313 Porto, Portugal; E-Mails: dpereira@ff.up.pt (D.M.P.); valentao@ff.up.pt (P.V.)

* Author to whom correspondence should be addressed; E-Mail: pandrade@ff.up.pt (P.B.A.); Tel.: +351-220-428-654; Fax: +351-226-093-390.

External Editor: Constantina Nasopoulou

Received: 11 October 2014; in revised form: 24 November 2014 / Accepted: 28 November 2014 / Published: 17 December 2014

\begin{abstract}
There is an increasing body of evidence of the positive impact of several marine lipids on human health. These compounds, which include $\omega-3$ polyunsaturated fatty acids, have been shown to improve blood lipid profiles and exert anti-inflammatory and cardioprotective effects. The high instability of these compounds to oxidative deterioration and their hydrophobicity have a drastic impact in their pharmacokinetics. Thus, the bioavailability of these compounds may be affected, resulting in their inability to reach the target sites at effective concentrations. In this regard, micro/nanoparticles can offer a wide range of solutions that can prevent the degradation of targeted molecules, increase their absorption, uptake and bioavailability. In this work we will present the options currently available concerning micro- and nanodelivery systems for marine lipids; with emphasis on micro/nanoparticles; such as micro/nanocapsules and emulsions. A wide range of bottom-up approaches using casein, chitosan, cyclodextrins, among others; will be discussed.
\end{abstract}

Keywords: fatty acids; $\omega-3$; liposomes; microparticles; nanoparticles; EPA; DHA

\section{Fatty Acids in Health \& Disease}

Fatty acids are lipophilic molecules that can be found in all living organisms. They can occur in their free form or, alternatively, integrate more complex lipids, such as triglycerides, phospholipids or glycolipids. In addition, fatty acids can be part of lipoproteins, lipopolysaccharides and alkaloids, among others. 
As it has been reviewed before [1], fatty acids exhibit a very diversified chemistry, which results in several possible structures that can be saturated/unsaturated, branched or cyclic, and with distinct functional groups, such as hydroxyl, keto and epoxy in some taxonomic groups.

Lipids in general, fatty acids in particular, serve three major roles in organisms: they are structural components of biological membranes, provide energy reserves and serve as biologically active molecules exerting a wide range of functions. Several epidemiological studies, as well as randomized and controlled trials, show that polyunsaturated fatty acids (PUFA), such as eicosapentaenoic acid (EPA) and docosahexaenoic acid (DHA), have benefic effects on cardiovascular diseases by lowering cardiovascular risk [2-6]. The mechanisms by which this effect is accomplished are diverse and include lowering of blood lipid levels, improved endothelial function and attenuated inflammatory responses [5,7].

Many inflammatory conditions result from an excessive production of pro-inflammatory mediators like eicosanoids, prostaglandin $\mathrm{E}_{2}\left(\mathrm{PGE}_{2}\right)$ and leukotriene $\mathrm{B} 4\left(\mathrm{LTB}_{4}\right)$. These molecules are synthesized in the organism from the $\omega-6$ FA arachidonic acid (C20:4 $\omega-6)$. If we consider that western diet results in high $\omega-6$ and low $\omega-3$ ratio of PUFA [8], it may be postulated that correction of these proportions, by increasing the consumption of $\omega$-3 FA like EPA (C20:5 $\omega-3)$ and DHA (C22:6 $\omega-3)$, may change this trend [9], as they may replace arachidonic acid as an eicosanoid substrate in cell membranes [10]. In addition, several works have shown that the anti-inflammatory properties of FA can also occur downstream of this process [11].

Regardless of the diversity of their biological effects, one of the factors that hinder the use of fatty acids in health and disease is related to their bioavailability.

In this regard, nano- and microsystems constitute important strategies for the delivery of these molecules, by providing both increased dissolution and protection against degradation [12-20].

\section{Nano- and Microencapsulation}

The general concept of encapsulation refers to a process by which a material (solid, liquid or gas) is packed within sphere-shaped structures. According to the particle size, microparticles or nanoparticles can be obtained, their applications being found across several areas, e.g., in food and pharmaceutical industries [21]. Particles in the 1-800 $\mu \mathrm{m}$ range are known as microparticles, microspheres or microcapsules, while others below $1 \mu \mathrm{m}$ are considered to be nanoparticles, nanospheres or nanocapsules [22], although some authors prefer to refer to nanoparticles only when their size is lower than $100 \mathrm{~nm}$.

Microencapsulation technology has initially been used almost exclusively in the pharmaceutical industry as a method for protecting sensitive components and, sometimes, as a way to increase drug delivery [23-25]. Given the potential of this approach, it has been extended to other industries, namely the food industry, as a strategy for protecting nutrients and delaying oxidation [26,27]. Microencapsulation is a process in which small particles of the active and/or sensitive component, such as fish oil or pure fatty acids, known as the core, are packaged within an encapsulating matrix [28].

Nanoparticles can be prepared by two basic approaches: either by a 'top-down"' approach, in which nanoparticles are produced by means of physical processing of several materials, or alternatively by a " 'bottom-up" approach, in which nanoparticles are produced via self-assembly and self-organization of smaller molecules [1]. Nowadays the general approach relies in both strategies to produce bioactive molecules-loaded nanoparticles that will result in improved availability in the human body. Thus, the 
efficacies of active ingredients, such as phytochemicals, vitamins, nutrients, or minerals, are preserved against the destructive conditions that oral consumption and absorption through the digestive track represent [13].

The differences between nano- and microparticles extend beyond the size, translating into their chemical and physical characteristics, which, in turn, have a marked impact in their application and potential use. An interesting example is that of microemulsions vs nanoemulsions.

Nanoemulsions are droplets of multiphase colloidal dispersions that are obtained from one liquid in another immiscible liquid, the chemical identity of the components and techniques used playing a pivotal role in the size of the droplets [29-32].

Several characteristics distinguish nanoemulsions from their micro- counterparts. From an optical point of view, nanoemulsions are frequently transparent owing to their size, which is smaller than visible wavelengths. Differently, microemulsions scatter visible light, thus originating a white opaque appearance [30]. Another interesting feature of nanoemulsions relies in their metastability, thus allowing them to be diluted with water without relevant changes in the distribution of droplet size [33].

In the specific case of marine $\omega-3$ fatty acids, one common drawback of their use and formulation is their hydrophobicity and the rate at which they oxidize, thus originating secondary lipids oxidation products that arise from the decomposition of hydroperoxides, mainly aldehydes, ketones and alcohols of distinct chain lengths and degrees of unsaturation, such as (Z)-1,5-octadiene-3-one, (E,Z)-2,4-heptadienal, 1-penten-3-ol and (Z)-4-heptenal [16,34]. Most of these compounds display low odor thresholds, thus affecting the sensory quality at very low concentrations $[16,35]$. For this reason, stabilization in aqueous medium and protection against stimuli that trigger deterioration is required. In the case of $\omega-3$ fatty acids and other components of fish oil, micro/nanoencapsulation is successfully used to protect against photo-oxidation and appearance of oxidized off-flavors, thus contributing to their quality and increased shelf-life [14-16,36]. By controlling the properties of the materials used and variables like $\mathrm{pH}$ and temperature, controlled release of the core materials can be achieved. In what concerns to stability, several factors besides composition are known to interfere, namely drying methods like spray granulation, spray drying and freeze drying [36].

From an experimental point of view, the rate of oxidation and formation of secondary lipids oxidation products is frequently evaluated by measuring lipid hydroperoxides, thiobarbituric acid reactive substances (TBARS) and headspace propanal/hexanal by gas chromatography- solid phase microextraction (GC-SPME).

\section{Case Studies: Micro- and Nanodelivery Systems for Fatty Acids}

\subsection{Lipidic Systems}

\subsubsection{Liposomes}

Liposomes are bilayer spherical structures constituted by surfactants, such as phospholipids (Figure 1). They are obtained by self-assembly of lipid molecules in aqueous systems, owing to their amphiphilic molecules, which adopt the bilayer sphere architecture as a way to shield the hydrophobic groups in their interior while maintaining the polar head groups in contact with the aqueous phase $[37,38]$. 
Figure 1. General structure of a liposome.

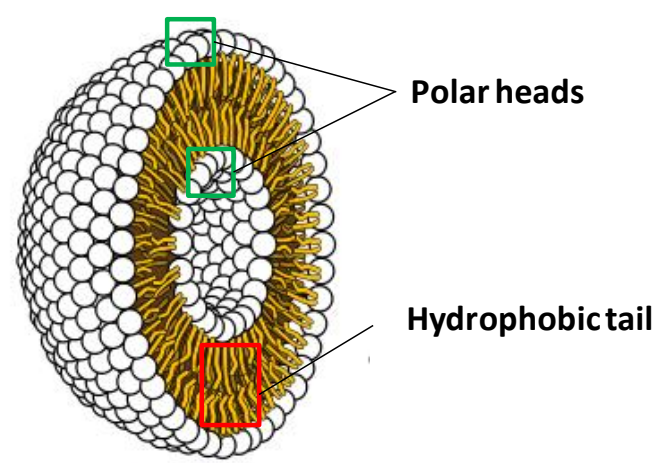

Owing to their chemical robustness, liposomes can be used to formulate, carry, deliver and release water-soluble, lipid-soluble and amphiphilic molecules [39-42].

From a physical point of view, liposomes can present sizes ranging from $20 \mathrm{~nm}$ to a few hundred $\mu \mathrm{m}$ [43]. Several factors, including stability, are markedly affected by intrinsic and extrinsic factors. As intrinsic factors we can refer the nature and identity of the liposome components and trapped molecules which, in turn, affect both the rheological properties and surface charge [44,45]. In the case of surface charge, its impact in electrostatic interactions is of pivotal importance in designing liposomes, due to its relevance in pharmacokinetics, for instance by affecting disintegration and hence the release profile of trapped compounds. Likewise, extrinsic factors, such as temperature, $\mathrm{pH}$ and ionic strength, have considerable influence in the chemical and physical stability of liposomes [46].

Temperature-sensitive liposomes can be produced by modifying the lipid layers with specific polymers that changes their solubility at a critical temperature, thus destabilizing the liposome membrane [30]. However, to the best of our knowledge, this approach has yet to be applied to liposomes with marine fatty acids.

In an interesting work, Cansell et al., used liposomes produced from an extract of marine lipids rich in $\omega-3$ PUFA as a delivery system for fatty acids supplements, thus increasing their bioavailability in rats [47]. The lipid profile of the animals was compared after feeding with regular fish oil and fish oil incorporated in the liposomes membranes. When using liposomes, fatty acid absorption was $98 \% \pm 1 \%$, while in the case of plain fish oil this value was reduced to $73 \% \pm 6 \%$. When studying individual compounds, DHA proportion in lymph was higher after liposome ingestion (78\%) than after fish oil ingestion (47\%). Interestingly, phospholipids concentration in lymph was not affected, regardless of the fat source, which is compatible with phospholipid regulation by de novo triacylglycerol (TAG) synthesis. An interesting result was that the distribution of $n$-3 PUFA esterified on the $s n-2$ position of chylomicron TAG depended on the lipid source administered.

This in vivo study confirmed that marine lipids are an interesting strategy for the delivery of PUFAs to the organism [47].

\subsubsection{Multi-Layered Emulsions}

Multi-layered emulsions constitute a robust strategy for protecting fish oils against oxidation. This is achieved by controlling each layer's composition, net electrical charge, permeability, and environmental 
responsiveness which, in turn, allows minimizing the exposure to temperature, mechanical agitation and $\mathrm{pH}$ [13].

Monodispersed fish oil-in-water emulsions have been laminated by applying several interfacial membranes in a layer-by-layer (LbL) approach [48]. While the primary emulsion was obtained through a membrane homogenizer using anionic citric acid ester of mono- and diglyceride, the second and tertiary emulsions relied on the electrostatic deposition of cationic chitosan and anionic sodium alginate, respectively, on the surfaces of the oil droplets. The positively charged secondary emulsions $(+56.27 \pm$ $2.5 \mathrm{mV})$ were more stable to lipid oxidation when compared to negatively charged primary $(-45.13 \pm$ $1.7 \mathrm{mV}$ ) and tertiary emulsions $(-24.8 \pm 1.2 \mathrm{mV})$, as evaluated by the levels of hydroperoxides, TBARS and headspace propanal/hexanal by GC-SPME. When evaluating the rate of digestion of oil droplets as a function of the different layers, lipid digestion was found to decrease with multilayer coating [48].

In another work, multilayer emulsifier systems comprising $\beta$-lactoglobulin and citrus/sugar beet pectin were tested [13]. Sugar beet pectin was used as it contains ferulic acid, thus being an antioxidant candidate for preventing oxidation of fish oil. Oil-in-water emulsions with $\beta$-lactoglobulin were prepared, upon which pectins were electrostatically deposited. Emulsions prepared with 1\% oil, $0.05 \%$ $\beta$-lactoglobulin and $0.06 \%$ pectins were physically stable for up to 16 days. Emulsions prepared with the multilayer system of $\beta$-lactoglobulin and citrus pectin were more stable than emulsions stabilized with $\beta$-lactoglobulin alone, as revealed by monitoring lipid hydroperoxide-derived propanal formation. Emulsions prepared with $\beta$-lactoglobulin plus sugar beet pectin were less stable than emulsions stabilized with $\beta$-lactoglobulin alone, despite the presence of ferulic acid in the sugar beet pectin [13].

The authors hypothesized that the presence of high levels of iron and copper could result in oxidative stress that surpassed the antioxidant capacity of ferulic acid [13].

In another study using a LbL approach (from menhaden oil) the primary layer was lecithin and the secondary one was chitosan-lecithin, microencapsulation being achieved by spray-drying with corn syrup [49]. In this system the interfacial properties of microencapsulated lecithin-chitosan multilayer emulsion droplets remained intact upon reconstitution into an aqueous system, the reconstituted secondary emulsion being more stable than the primary one. More physically stable powders were obtained when high amounts of corn syrup (10\%-20\%) were used [49].

\subsubsection{Cochleates}

Cochleates are a lipid-based system obtained from the precipitation of negatively charged lipids and cations [50].

One of the most widespread production processes is the crystallization of phospholipids from soy in the presence of calcium. From a structural point of view, these particles comprise phospholipid bilayers stacked as sheets and rolled in a spiral configuration, with solutions of multivalent cations locating between each sheet, "wrapping" around oil droplets containing the molecules to be trapped (Figure 2).

Experimentally, these particles can be obtained by slow introduction of cations into suspensions of anionic liposomes, thus causing the liposomes to fuse together, cochleate formation being indicated by a rapid increase of turbidity [51]. As reviewed by Loveday et al., small unilamellar liposomes prepared by film hydration give more uniform cigar-shaped cochleates than multilamellar liposomes from powdered phospholipids [52]. Differently, in the hydrogel-based method, liposomes are mixed with a 
polymer and injected into a solution of a second, non-miscible polymer, e.g., dextran and polyethylene glycol (PEG). Calcium is added to the water-in-water emulsion and diffuses slowly from the PEG continuous phase into the dispersed dextran-liposome phase, producing nanocochleates [53]. Sub-micrometre cochleates can be produced by this method, whereas the trapping method gives larger cochleates.

Figure 2. Schematic representation of nanocochleates.

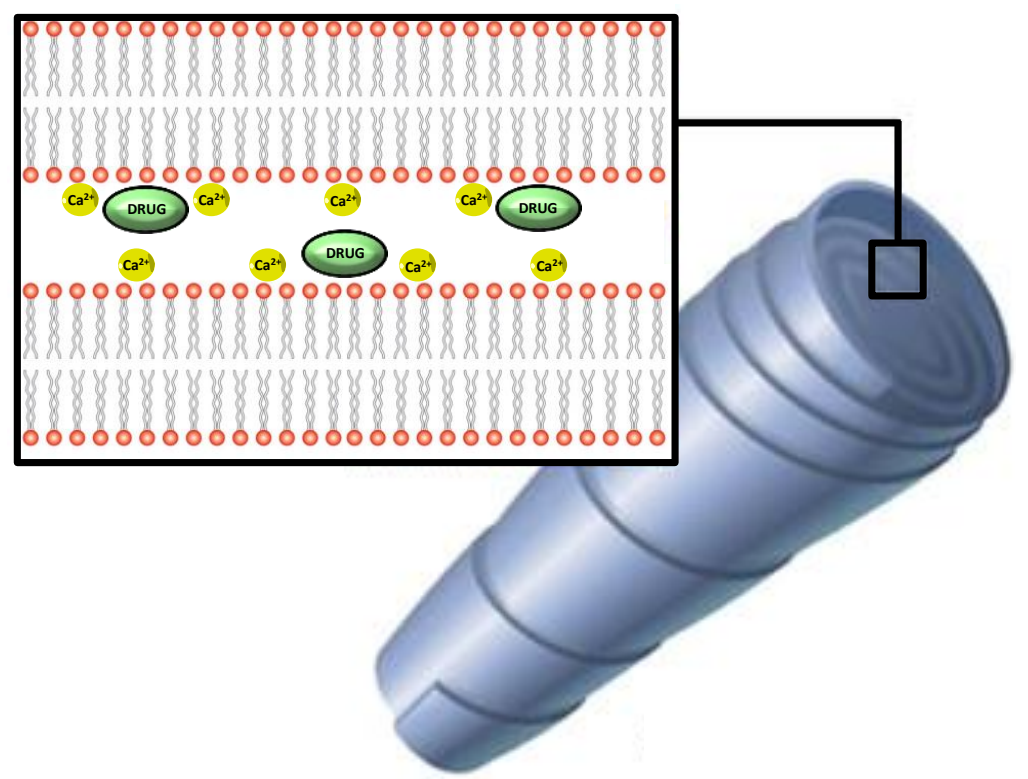

At least one product using this technology is currently being marketed as a tool for protecting $\omega$-3 fatty acids, with claims upon enhanced stability BioGeode $^{\mathrm{TM}}$, by BioDelivery Sciences International, Inc., Raleigh, NC, USA).

\subsubsection{Simple Emulsion-Based Systems}

Fish oil was has been microencapsulated using a matrix consisting of sugar beet pectin and glucose syrup [54]. Several experimental conditions were evaluated and median oil droplet size was shown to be markedly affected by the composition of the emulsion, as well as by the homogenization pressure. The best results were obtained when the emulsion was produced with $50 \%$ oil and around $2 \%$ of sugar beet pectin, yielding droplet size below $2 \mu \mathrm{m}$ and maximum viscosity of $179 \mathrm{mPa}$ [54].

An encapsulation technique for tuna oil using an ultrasonic atomizer and a process workflow comprising three steps (emulsification, ultrasonic atomization and freeze drying) has been reported [55]. Distinct wall materials, such as chitosan, maltodextrin and whey protein isolate were tested, together with their respective proportions. Chitosan in combination with maltodextrin yielded the particles with the smallest size and the highest emulsion stability. Gas chromatography-flame ionization detection (GC-FID) studies have assessed the effect of encapsulation on the fatty acids profile of fish oil. Prior to encapsulation, saturated fatty acids and PUFA were major compounds. Encapsulation led to EPA and DHA levels of $24 \mathrm{~g} / 100 \mathrm{~g}$, with simultaneous increase of the levels of the monounsaturated fatty acid (MUFA) eicosenoic acid [55]. EPA and DHA content of the encapsulated fish oil was higher than the commercial specification $(10 \mathrm{~g} / 100 \mathrm{~g})$ [55]. 
Nanocapsules of $\alpha$-linolenic acid have been produced through a modified emulsion diffusion technique [56]. Several conditions were tested, namely the use of polylactic acid as the encapsulating polymer, acetone and ethyl acetate as organic solvents and also tween 20, gelatin and Pluronic-F68 as stabilizers. When comparing all conditions, acetone revealed to be better than ethyl acetate, while Tween 20 exceeded Pluronic-F68. For the formation of smaller nanocapsules, an organic to aqueous phase ratio of 1:5 was the most suitable. Parameters, such as particle size and zeta potential, were monitored, values in the range of $100 \mathrm{~nm}$ and $+33 \mathrm{mV}$ being obtained [56].

\subsection{Non-Lipidic Systems}

\subsubsection{Cyclodextrins}

Cyclodextrins are a group of cyclic oligosaccharides that can be obtained from starch by enzymatic conversion. Two main topologies of native cyclodextrins can be found: crystalline and aqueous solution. Either channel-type and cage-type aggregates can be obtained in crystal structures [57]. Several parameters are known to influence the cyclodextrin size, including molar mass, concentration and host:guest molecule ratio [58]. Several derivatives exist, namely $\alpha$-, $\beta$ - and $\gamma$-cyclodextrin, which are 6 , 7 and -8 membered, respectively (Figure 3).

Figure 3. Chemical and toroid structure of cyclodextrins.
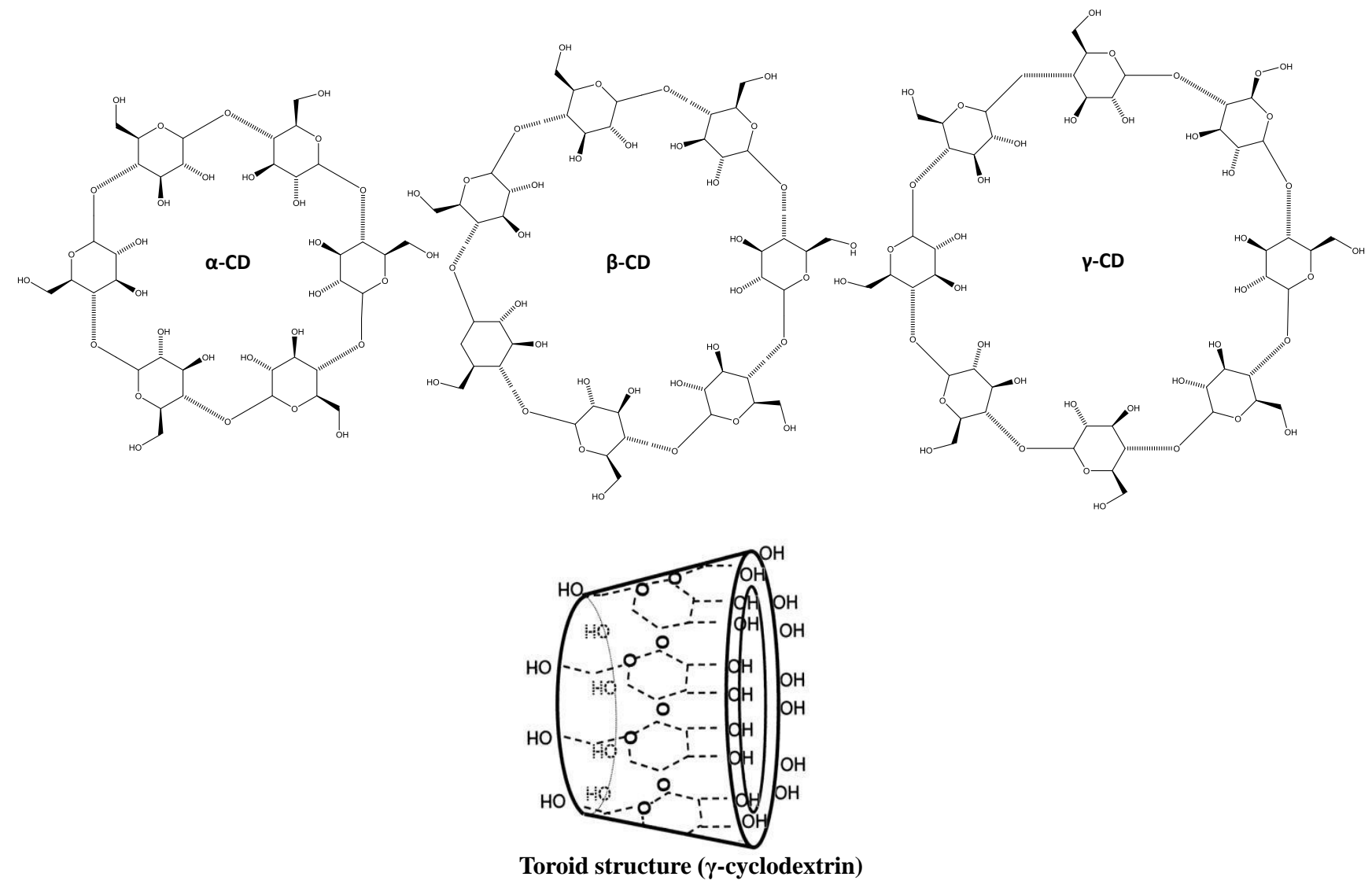
There is a wide range of applications for cyclodextrins across several industries, namely pharmaceutical, food and cosmetic, mostly due to their low cost and toxicity. In addition, cyclodextrins are regarded as excellent options for masking odors and off-flavors [16].

Encapsulation involving cyclodextrins relies in selective molecular combination of host and guest molecules, ultimately yielding supramolecular systems [21].

Due to the chemical and physical properties of cyclodextrins, fatty acids and their methyl-, ethyl and saccharose esters, monoglycerides and similar long apolar alkane chain-bearing molecules, are regarded as good complex-forming guest molecules [17].

In what concerns to the use of cyclodextrins as carriers for $\omega-3$ fatty acids, the authors report the influence of the $\beta$-cyclodextrins-fish oil ratio in several parameters. Among the several conditions tested, the ratio 10:20 was the one that scored best regarding encapsulation efficiency $(84.1 \%)$, fish oil loading $(62.7 \%)$, leakage after freeze-drying (11.0\%) and EPA encapsulation efficiency (6.5\%). After 3 days, freeze-dried $\beta$-cyclodextrins-fish oil retained $97 \%$ of fish oil within the particles [12]. The size of these particles was highly dependent on the fish oil loading, ranging from 250 to $700 \mathrm{~nm}$, this last resulting from $\beta$-cyclodextrins:fish oil ratio of 10:20.

In another study cyclodextrins were used in combination with whey protein concentrate [59]. When evaluating several analogues of the cyclodextrins series, the $\gamma$ - homologue scored better than $\beta$-cyclodextrin regarding emulsion stability and encapsulation efficiency. Whey protein concentrate:cyclodextrin ratio was the main factor affecting particle size, which ranged from 120 to $700 \mathrm{~nm}$. Reduction of odor in the $70 \%$ range was also reported, as well as oxidative stability, as assessed by peroxide values [59].

\subsubsection{Caseins}

Caseins are the major milk proteins, in which they occur in the form of large colloidal particles, casein micelles (Figure 4), presenting an average diameter of $150 \mathrm{~nm}$. There are four main types of casein ( $\alpha \mathrm{s} 1$, $\alpha$ s, $\beta$ - and $\kappa$-casein), all of them being held together via hydrophobic and electrostatic interactions [38].

Figure 4. Structural arrangement of casein micelles. CP-Calcium phosphate.

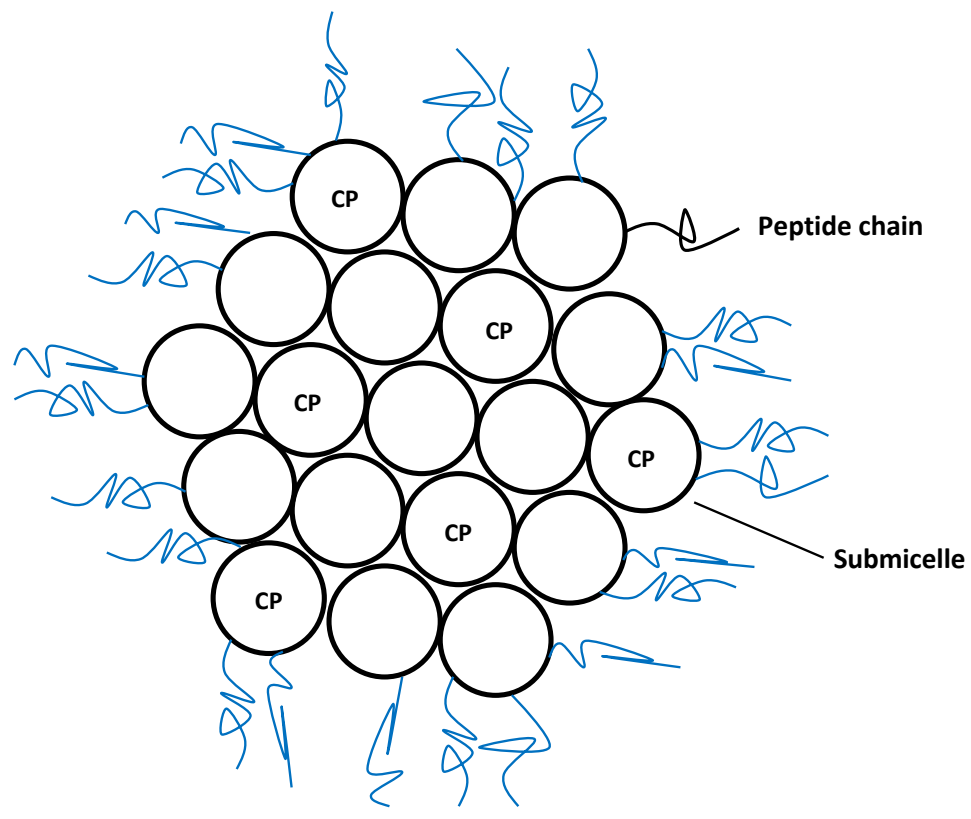


Experimentally, caseins are usually prepared by isoelectric precipitation of skim milk at $\mathrm{pH}$ lower than 5, with subsequent resolubilization with alkaline salts using calcium, potassium, sodium, or magnesium hydroxide to increase the $\mathrm{pH}$ to 6.7 and spray-drying to originate caseinates [60]. Structurally, the resulting micelles are composed of calcium phosphate clusters together with different casein molecules from the above mentioned families.

From a technological point of view, the most relevant characteristic of micelles, including caseins, is their ability to host non-polar molecules, hence improving their solubilization and increasing their bioavailability. For this reason, nowadays casein micelles are used for nano-encapsulation and stabilization of several components, notably in the food industry, where they are used to incorporate hydrophobic molecules in non-fat or low-fat products [38].

Caseins have been used to entrap and deliver DHA, one of the most important marine lipids. Each molecule of casein binds 3-4 DHA molecules, the resulting micelles displaying an average size of 50-60 $\mathrm{nm}$ when prepared at low temperatures in the presence of calcium and phosphate. Good colloidal stability and protective effect against DHA oxidation is observed at $4{ }^{\circ} \mathrm{C}$ [20].

In a different approach, caseinate was complexed with pectin in order to obtain biopolymer-based hydrogel microspheres, which were used to encapsulate fish oil [61]. This method has shown to improve the stability of fish oil concerning to lipid oxidation, as assessed by the low levels of hydroperoxides up to 7 days of storage. From a mechanistic point of view, this is believed to be a consequence of a high local concentration of antioxidant protein around the emulsified lipids [61].

\subsubsection{Zein Nanoparticles}

Zein are a group of water-insoluble prolamine storage proteins that are mainly present in the endosperm of corn kernels [62]. Due to their organoleptic characteristics (odorless and tasteless), this material is a promising tool for carrying ingredients in delivery systems. Zein comprises at least four types of proteins, namely $\alpha-, \beta-, \gamma-$, and $\delta$-zein, each with different molecular weights and solubility. Industrially, commercial zein is obtained from corn gluten meal, a co-product of corn wet milling [63].

Several studies can be found addressing the use of zein as a carrier biopolymer for water-soluble molecules, such as heparin and ivermectin [64,65]. Concerning to marine fatty acids, fish oil has been encapsulated in zein particles in the nanometer range, namely $350-450 \mathrm{~nm}$. Following freeze-drying, good oxidative stability was found for samples with zein-oil ratio of 4:1 or lower [66].

In another work using DHA instead of whole fish oil, Torres-Giner et al. used an ultrathin zein encapsulation approach [18]. This strategy revealed to increase the stability of DHA by reducing its degradation rate by around 2.5 fold and yielding substantially lower off-flavor content.

The digestion of DHA in fish oil encapsulated in porous starch granules coated with zein in the gastrointestinal tract of rats has also been addressed [19]. Initial in vitro studies showed that zein could be decomposed by proteolytic enzymes and in vivo experiments showed that particles loaded with fish/coconut/corn oil lowered serum triglycerides and phospholipids in rats. In the fish oil group the proportion of DHA in total fatty acids was significantly higher in both liver and brain. These results indicate that fish oil encapsulated in porous starch granules coated with zein are digested and absorbed [19]. 


\section{Conclusions}

An increasing number of works describe delivery systems that aim to increase the bioavailability of fatty acids and to protect them from oxidation, thus enhancing their absorption. In this regard, several systems, comprising distinct particle architectures, have been developed. While it is expected that in the next few years many advances in this field are likely to occur, some topics still require further attention.

In particular, it is important to address the in vivo bioavailability and pharmacokinetics of these systems, as many of the new micro- and nanoparticles have not yet been evaluated in this regard, only few works reaching the in vivo stage.

Despite the potential and academic interest of many of the systems discussed herein, when addressing this topic from an industrial perspective, some considerations should be taken into account. Regardless of the multiple advantages of carbohydrate and protein-based micro-/nanoparticles, they may hinder full scale-up due to technical requirement in terms of chemical or heat treatments. On the other hand, lipid-based particles may be easier to process at an industrial level and may pose advantages regarding encapsulation efficiency and toxicity.

Yet, another subject that requires attention is related with the lack of homogeneity regarding the parameters that are presented when characterizing micro/nanoparticles. For example, many works do not present data concerning to stability, the presence of oxidation products or encapsulation efficiency, which hinders the direct comparison of different techniques.

\section{Conflicts of Interest}

The authors declare no conflict of interest.

\section{References}

1. Pereira, D.M.; Vinholes, J.; Correia-da-Silva, G.; Valentao, P.; Teixeira, N.; Andrade, P.B. Fatty acids in marine organisms: In the pursuit of bioactive agents. Curr. Pharm. Anal. 2011, 7, 108-119.

2. Balk, E.M.; Lichtenstein, A.H.; Chung, M.; Kupelnick, B.; Chew, P.; Lau, J. Effects of omega-3 fatty acids on serum markers of cardiovascular disease risk: A systematic review. Atherosclerosis 2006, 189, 19-30.

3. Hooper, L.; Thompson, R.L.; Harrison, R.A.; Summerbell, C.D.; Ness, A.R.; Moore, H.J.; Worthington, H.V.; Durrington, P.N.; Higgins, J.; Capps, N.E. Risks and benefits of omega 3 fats for mortality, cardiovascular disease, and cancer: Systematic review. BMJ 2006, 332, 752-760.

4. Hu, F.B.; Bronner, L.; Willett, W.C.; Stampfer, M.J.; Rexrode, K.M.; Albert, C.M.; Hunter, D.; Manson, J.E. Fish and omega-3 fatty acid intake and risk of coronary heart disease in women. JAMA 2002, 287, 1815-1821.

5. Kris-Etherton, P.M.; Harris, W.S.; Appel, L.J. Fish consumption, fish oil, omega-3 fatty acids, and cardiovascular disease. Circulation 2002, 106, 2747-2757.

6. Kris-Etherton, P.M.; Harris, W.S.; Appel, L.J. Omega-3 fatty acids and cardiovascular disease new recommendations from the American Heart Association. Arterioscl. Throm. Vas. 2003, 23, 151-152.

7. Calder, P.C. $n$-3 Polyunsaturated fatty acids, inflammation, and inflammatory diseases. Am. J. Clin. Nutr. 2006, 83, 1505S-1519S. 
8. Simopoulos, A.P. Importance of the ratio of omega-6/omega-3 essential fatty acids: Evolutionary aspects. World Rev. Nutr. Diet. 2003, 92, 1-22.

9. Wall, R.; Ross, R.P.; Fitzgerald, G.F.; Stanton, C. Fatty acids from fish: The anti-inflammatory potential of long-chain omega-3 fatty acids. Nutr. Rev. 2010, 68, 280-289.

10. Simopoulos, A.P. Essential fatty acids in health and chronic diseases. Forum Nutr. 2003, 56, 67-70.

11. Pereira, D.M.; Valentão, P.; Andrade, P.B. Lessons from the sea: Distribution, SAR and molecular mechanisms of anti-inflammatory drugs from marine organisms. In Studies in Natural Products Chemistry (Bioactive Natural Products); Atta-ur-Rahman, Ed.; Elsevier Science Publishers: Amsterdam, The Netherlands, 2013.

12. Choi, M.-J.; Ruktanonchai, U.; Min, S.-G.; Chun, J.-Y.; Soottitantawat, A. Physical characteristics of fish oil encapsulated by $\beta$-cyclodextrin using an aggregation method or polycaprolactone using an emulsion-diffusion method. Food Chem. 2010, 119, 1694-1703.

13. Katsuda, M.S.; McClements, D.J.; Miglioranza, L.H.S.; Decker, E.A. Physical and oxidative stability of fish oil-in-water emulsions stabilized with $\beta$-lactoglobulin and pectin. J. Agric. Food Chem. 2008, 56, 5926-5931.

14. Kolanowski, W.; Jaworska, D.; Weißbrodt, J.; Kunz, B. Sensory assessment of microencapsulated fish oil powder. J. Am. Oil Chem. Soc. 2007, 84, 37-45.

15. Kolanowski, W.; Ziolkowski, M.; Weißbrodt, J.; Kunz, B.; Laufenberg, G. Microencapsulation of fish oil by spray drying-impact on oxidative stability. Part 1. Eur. Food Res. Technol. 2006, 222, 336-342.

16. Serfert, Y.; Drusch, S.; Schwarz, K. Sensory odour profiling and lipid oxidation status of fish oil and microencapsulated fish oil. Food Chem. 2010, 123, 968-975.

17. Szente, L.; Szejtli, J.; Szemán, J.; Kató, L. Fatty acid-cyclodextrin complexes: Properties and applications. J. Incl. Phenom. Macro. 1993, 16, 339-354.

18. Torres-Giner, S.; Martinez-Abad, A.; Ocio, M.J.; Lagaron, J.M. Stabilization of a nutraceutical omega-3 fatty acid by encapsulation in ultrathin electrosprayed zein prolamine. J. Food Sci. 2010, 75, N69-N79.

19. Xu, X.; Hasegawa, N.; Doi, U.; Umekawa, H.; Takahashi, T.; Suzuki, K.; Furuichi, Y. Biological availability of docosahexaenoic acid from fish oil encapsulated in zein-coated porous starch granules in rats. Food Sci. Technol. Res. 2000, 6, 87-93.

20. Zimet, P.; Rosenberg, D.; Livney, Y.D. Re-assembled casein micelles and casein nanoparticles as nano-vehicles for $\omega-3$ polyunsaturated fatty acids. Food Hydrocoll. 2011, 25, 1270-1276.

21. Choi, M.-J.; Ruktanonchai, U.; Soottitantawat, A.; Min, S.-G. Morphological characterization of encapsulated fish oil with $\beta$-cyclodextrin and polycaprolactone. Food. Res. Int. 2009, 42, 989-997.

22. Kwak, H.-S. Nano- and Microencapsulation for Foods; Wiley-Blackwell: Oxford, UK, 2014.

23. Ahmad, M.; Madni, A.; Usman, M.; Munir, A.; Akhtar, N.; Khan, H.S. Pharmaceutical micro encapsulation technology for development of controlled release drug delivery systems. WASET 2011, 75, 384-387.

24. Lam, P.; Gambari, R. Advanced progress of microencapsulation technologies: In vivo and in vitro models for studying oral and transdermal drug deliveries. J. Control. Release 2014, 178, 25-45. 
25. Singh, M.; Hemant, K.; Ram, M.; Shivakumar, H. Microencapsulation: A promising technique for controlled drug delivery. Res. Pharm. Sci. 2010, 5, 65-77.

26. Garg, M.; Wood, L.; Singh, H.; Moughan, P. Means of delivering recommended levels of long chain n-3 polyunsaturated fatty acids in human diets. J. Food Sci. 2006, 71, R66-R71.

27. Taneja, A.; Singh, H. Challenges for the delivery of long-chain $n-3$ fatty acids in functional foods. Annu. Rev. Food Sci. T. 2012, 3, 105-123.

28. Desai, K.G.H.; Jin Park, H. Recent developments in microencapsulation of food ingredients. Dry Technol. 2005, 23, 1361-1394.

29. Anton, N.; Benoit, J.-P.; Saulnier, P. Design and production of nanoparticles formulated from nano-emulsion templates-A review. J. Control. Release 2008, 128, 185-199.

30. Fathi, M.; Mozafari, M.; Mohebbi, M. Nanoencapsulation of food ingredients using lipid based delivery systems. Trends Food Sci. Tech. 2012, 23, 13-27.

31. Shakeel, F.; Ramadan, W. Transdermal delivery of anticancer drug caffeine from water-in-oil nanoemulsions. Colloid Surf. B 2010, 75, 356-362.

32. Talegaonkar, S.; Mustafa, G.; Akhter, S.; Iqbal, Z. Design and development of oral oil-in-water nanoemulsion formulation bearing atorvastatin: In vitro assessment. J. Disper. Sci. Technol. 2010, $31,690-701$.

33. Gutiérrez, J.; González, C.; Maestro, A.; Sole, I.; Pey, C.; Nolla, J. Nano-emulsions: New applications and optimization of their preparation. Curr. Opin. Colloid Interface Sci. 2008, 13, 245-251.

34. Let, M.B.; Jacobsen, C.; Frankel, E.N.; Meyer, A.S. Oxidative flavour deterioration of fish oil enriched milk. Eur. J. Lipid Sci. Tech. 2003, 105, 518-528.

35. Frankel, E.N. Lipid Oxidation; Oily Press Lipid Library Series, Woodhead Publishing Limited: Cambridge, UK, 2005.

36. Anwar, S.H.; Kunz, B. The influence of drying methods on the stabilization of fish oil microcapsules: Comparison of spray granulation, spray drying, and freeze drying. J. Food Eng. 2011, 105, 367-378.

37. Arunagirinathan, G.H.S.M.; Bellare, J.R. Self-assembled surfactant nano-structures important in drug delivery: A review. Indian J. Exp. Biol. 2007, 45, 133-159.

38. Kaya-Celiker, H.; Mallikarjunan, K. Better nutrients and therapeutics delivery in food through nanotechnology. Food. Eng. Rev. 2012, 4, 114-123.

39. Akbarzadeh, A.; Rezaei-Sadabady, R.; Davaran, S.; Joo, S.W.; Zarghami, N.; Hanifehpour, Y.; Samiei, M.; Kouhi, M.; Nejati-Koshki, K. Liposome: Classification, preparation, and applications. Nanoscale Res. Lett. 2013, 8, 102.

40. Kuznetsova, N.R.; Sevrin, C.; Lespineux, D.; Bovin, N.V.; Vodovozova, E.L.; Mészáros, T.; Szebeni, J.; Grandfils, C. Hemocompatibility of liposomes loaded with lipophilic prodrugs of methotrexate and melphalan in the lipid bilayer. J. Control. Release 2012, 160, 394-400.

41. Maherani, B.; Arab-Tehrany, E.; Mozafari, M.R.; Gaiani, C.; Linder, M. Liposomes: A review of manufacturing techniques and targeting strategies. Curr. Nanosci. 2011, 7, 436-452.

42. Mufamadi, M.S.; Pillay, V.; Choonara, Y.E.; du Toit, L.C.; Modi, G.; Naidoo, D.; Ndesendo, V.M. A review on composite liposomal technologies for specialized drug delivery. J. Drug Deliv. 2011, 2011, doi:10.1155/2011/939851. 
43. Chen, H.; Weiss, J.; Shahidi, F. Nanotechnology in nutraceuticals and functional foods. Food Technol. 2006, 60, 30-36.

44. Alves, M.P.; Raffin, R.P.; Fagan, S.B. Rheological behavior of semisolid formulations containing nanostructured systems. In Nanocosmetics and Nanomedicines; Springer: Berlin Heidelberg, Germany, 2011; pp. 37-45.

45. Lu, T.; Zhao Wang, Y.M.; Zhang, Y.; Chen, T. Influence of polymer size, liposomal composition, surface charge, and temperature on the permeability of $\mathrm{pH}$-sensitive liposomes containing lipid-anchored poly (2-ethylacrylic acid). Int. J. Nanomed. 2012, 7, 4917-4926.

46. Taylor, T.M.; Weiss, J.; Davidson, P.M.; Bruce, B.D. Liposomal nanocapsules in food science and agriculture. Crit. Rev. Food Sci. Nutr. 2005, 45, 587-605.

47. Cansell, M.; Nacka, F.; Combe, N. Marine lipid-based liposomes increase in vivo FA bioavailability. Lipids 2003, 38, 551-559.

48. Gudipati, V.; Sandra, S.; McClements, D.J.; Decker, E.A. Oxidative stability and in vitro digestibility of fish oil-in-water emulsions containing multilayered membranes. J. Agric. Food Chem. 2010, 58, 8093-8099.

49. Shaw, L.A.; McClements, D.J.; Decker, E.A. Spray-dried multilayered emulsions as a delivery method for $\omega-3$ fatty acids into food systems. J. Agric. Food Chem. 2007, 55, 3112-3119.

50. Zarif, L.; Graybill, J.R.; Perlin, D.; Mannino, R.J. Cochleates: new lipid-based drug delivery system. J. Liposome Res. 2000, 10, 523-538.

51. Evans, C.C.; Zasadzinski, J. Encapsulating vesicles and colloids from cochleate cylinders. Langmuir 2003, 19, 3109-3113.

52. Zarif, L. Drug delivery by lipid cochleates. Methods Enzymol. 2005, 391, 314-329.

53. Santangelo, R.; Paderu, P.; Delmas, G.; Chen, Z.-W.; Mannino, R.; Zarif, L.; Perlin, D.S. Efficacy of oral cochleate-amphotericin B in a mouse model of systemic candidiasis. Antimicrob. Agents Chemother. 2000, 44, 2356-2360.

54. Drusch, S. Sugar beet pectin: A novel emulsifying wall component for microencapsulation of lipophilic food ingredients by spray-drying. Food Hydrocoll. 2007, 21, 1223-1228.

55. Klaypradit, W.; Huang, Y.-W. Fish oil encapsulation with chitosan using ultrasonic atomizer. LWT_Food Sci. Technol. 2008, 41, 1133-1139.

56. Habib, S.M.; Amr, A.S.; Hamadneh, I.M. Nanoencapsulation of alpha-linolenic acid with modified emulsion diffusion method. J. Am. Oil Chem. Soc. 2012, 89, 695-703.

57. Saenger, W.; Jacob, J.; Gessler, K.; Steiner, T.; Hoffmann, D.; Sanbe, H.; Koizumi, K.; Smith, S.M.; Takaha, T. Structures of the common cyclodextrins and their larger analogues beyond the doughnut. Chem. Rev. 1998, 98, 1787-1802.

58. Loftsson, T.; Masson, M.; Brewster, M.E. Self-association of cyclodextrins and cyclodextrin complexes. J. Pharm. Sci.-US 2004, 93, 1091-1099.

59. Na, H.-S.; Kim, J.-N.; Kim, J.-M.; Lee, K.-Y. Encapsulation of fish oil using cyclodextrin and whey protein concentrate. Biotechnol. Bioproc. E. 2011, 16, 1077-1082.

60. Dickinson, E.; Singh, H. Protein functionality and aggregation. Milk protein functionality in food colloids. In Food Colloids: Interactions, Microstructure and Processing; Royal Society of Chemistry: Cambridge, UK, 2005. 
61. Zhang, Z.; Decker, E.A.; McClements, D.J. Encapsulation, protection, and release of polyunsaturated lipids using biopolymer-based hydrogel particles. Food Res. Int. 2014, 64, 520-526.

62. Shukla, R.; Cheryan, M. Zein: The industrial protein from corn. Ind. Crop Prod. 2001, 13, 171-192.

63. Zhu, F.; Kale, A.V.; Cheryan, M. Fractionation of zein by size exclusion chromatography. J. Agric. Food Chem. 2007, 55, 3843-3849.

64. Liu, X.; Sun, Q.; Wang, H.; Zhang, L.; Wang, J.-Y. Microspheres of corn protein, zein, for an ivermectin drug delivery system. Biomaterials 2005, 26, 109-115.

65. Wang, H.-J.; Lin, Z.-X.; Liu, X.-M.; Sheng, S.-Y.; Wang, J.-Y. Heparin-loaded zein microsphere film and hemocompatibility. J. Control. Release 2005, 105, 120-131.

66. Zhong, Q.; Tian, H.; Zivanovic, S. Encapsulation of fish oil in solid zein particles by liquid-liquid dispersion. J. Food Process. Pres. 2009, 33, 255-270.

(C) 2014 by the authors; licensee MDPI, Basel, Switzerland. This article is an open access article distributed under the terms and conditions of the Creative Commons Attribution license (http://creativecommons.org/licenses/by/4.0/). 